\title{
Impact of malocclusion on oral health related quality of life in young people
}

\author{
Yaghma Masood ${ }^{1}$, Mohd Masood ${ }^{2}$, Nurul Nadiah Binti Zainul ${ }^{3}$, Nurhuda Binti Abdul Alim Araby ${ }^{3}$, \\ Saba Fouad Hussain ${ }^{4}$ and Tim Newton ${ }^{5}$
}

\begin{abstract}
Background: The objectives for this study were to assess Oral Health Related Quality of Life (OHRQoL) in young people aged 15-25 who sought orthodontic treatment, and to measure the association between orthodontic treatment need (using the IOTN), sex, age and education level, and oral health related quality of life (OHRQoL).

Methods: Survey of a consecutive series of 323 young adults aged 15 to 25 years, attending orthodontic clinics at the Faculty of Dentistry, Universiti Teknologi MARA. Participants completed the Oral Health Impact Profile-14 (OHIP-14) and had a clinical examination including the Index of Orthodontic Treatment Need- Dental Health Component (IOTN-DHC). Data analyses included descriptive statistics, One-way ANOVA and bivariate and multivariate regression models.

Results: The mean overall score ( \pm SD) for OHIP-14 in young people aged 15-25 was $22.6 \pm 12.5$. The psychological discomfort domain was the domain where highest impact was recorded with a mean ( \pm SD) of $4.0 \pm 1.9$. The regression analyses showed a significant association of IOTN-DHC with overall OHIP-14 score $(p<0.05)$. Although females reported a slightly higher impact than males, this was not significant in both bivariate and multivariate analyses. Age group had a significant negative association with overall OHIP-14 score $(p<0.05)$. The $15-18$ year old group showed the highest impact on their quality of life due to malocclusion. Participants with a university education report a significantly higher impact on OHRQoL as compared to participants with only secondary education.
\end{abstract}

Conclusion: Malocclusion has a significant negative impact on OHRQOL and its domains. This is greatest for the psychological discomfort domain. Younger people and those with a university education report higher levels of impact. There was no reported difference in impact between male and females.

Keywords: Oral health related quality of life, Malocclusion, Treatment need, OHIP

\section{Background}

The concept of Oral Health-Related Quality of Life (OHRQoL) corresponds to the impact of oral health or disease on an individual's daily functioning, well-being or overall quality of life [1]. Conditions affecting oral health, including malocclusion, are highly prevalent, and have consequences not only for physical and economical well being, but can also impair quality of life by affecting function, appearance, interpersonal relationships, socializing, self-esteem and psychological well-being [2]. Research on the physical, social, and psychological impact of malocclusion on OHRQoL sheds light on the effects of malocclusion on people's lives and provides a greater

\footnotetext{
* Correspondence: drmasoodmohd@gmail.com

${ }^{2}$ Centre of Studies for Community Dentistry, Faculty of Dentistry, Universiti

Teknologi MARA, Shah Alam, Malaysia

Full list of author information is available at the end of the article
}

understanding of the demand for orthodontic treatment beyond the measurement of clinical parameters. In addition, since social and psychological effects are often the key motives for seeking orthodontic treatment, OHRQoL can be considered the best measurement for orthodontic treatment need and outcome [2]. Such research may be of great value to researchers, health planners, and oral health care providers [3].

Malocclusion differs from the majority of medical and dental conditions in that it is 'a set of dental deviations' rather than a disease, and orthodontic treatment does not cure a condition but rather corrects variations from an arbitrary norm [4]. This has led to debate about defining the point at which the extent of variation means that orthodontic treatment is desirable [5]. Further it has been suggested that the majority of oral health measures 
developed in dentistry are not applicable to orthodontic patients because most malocclusions are asymptomatic and related to esthetic challenges, as opposed to loss of function [4,6]. Additionally, a malocclusion can be perceived differently by the affected person, and a person's degree of awareness of their malocclusion might not be related to its severity [3]. Therefore, when evaluating the impact of a malocclusion, it is important to consider the different domains that can be affected and their relationships to the severity of malocclusion. Some people with a severe malocclusion are satisfied with or indifferent to their dental esthetics, whereas others are concerned about minor irregularities [3].

Need assessment for orthodontic treatment in dental clinics is traditionally normatively assessed using measures such as the Index of Orthodontic Treatment Need (IOTN $[3,7]$. However there is evidence that many adolescents with normative orthodontic treatment need measured by IOTN experience no impacts on their OHRQoL $[8,9]$. Therefore, the use of IOTN alone to establish orthodontic treatment need may be potentially problematic since some patients who have no psychosocial need for treatment would be perceived as requiring treatment [10,11]. The use of OHRQoL measures in addition to professional indices offers a potentially useful combination [7,12]. OHIP-14 can be used as a potential proxy measure to replace subjective clinical opinions for determining treatment need in youth [13].

Previous research exploring the relationship between malocclusions and OHRQoL, as well as the impact of orthodontic treatment on OHRQOL has been equivocal. Some authors found a strong relationship between malocclusion or orthodontic treatment need and OHRQoL $[12,14,15]$, but others reported no clear relationship $[4,16,17]$. Therefore, this study was conducted to assess OHRQoL in young people aged 15 to 25 years who sought orthodontic treatment in the Department of Orthodontics at Universiti Teknologi MARA Malaysia and to measure the association between orthodontic treatment need (using the IOTN), sex, age and education level, and OHRQoL.

\section{Methods}

\section{Study design}

Participants in the study were children and young adults aged 15 to 25 years, attending orthodontic clinics at the Faculty of Dentistry, Universiti Teknologi MARA, in Malaysia. The orthodontic clinic at Faculty of Dentistry UiTM is the biggest orthodontic clinic in Shah Alam city and nearby areas. It is a government clinic and all the patients who are judged to have a clinical need or who request for orthodontic treatment are referred to the Orthodontic clinic at Faculty of Dentistry UiTM.

All children and young adults aged 15 to 25 years, who either self-referred or were referred by their general dental practitioners to the orthodontic clinics at the Faculty of Dentistry, Universiti Teknologi MARA, in Malaysia. Most participants were motivated by their parents to seek orthodontic consultation. A convenience consecutive sampling approach was used. The participants were recruited at their first visit for orthodontic screening before starting any orthodontic treatment. The participants or where appropriate their parents were fully informed of the nature of the study and signed a consent form agreeing to participate in the study. To be eligible, the participant had to be in good general health. Participants who required a surgical intervention or who had chronic medical conditions, had received previous orthodontic treatment, had severe dentofacial anomalies such as cleft lip and palate, untreated dental caries, or poor periodontal health status as indicated by a community periodontal index score of 3 or more were excluded. This was to prevent possible confounding effects of these conditions on the participants' quality of life and to achieve a homogeneous group population. The Universiti Teknologi MARA (UiTM) Research Ethics Board approved all study procedures.

\section{Outcome variable (OHIP-14)}

OHRQoL was measured using a Malay language translated version of the 14-item Oral Health Impact Profile (OHIP14). It has been shown that the OHIP-14 has good reliability, validity, and precision $[18,19]$. The Malay version of OHIP-14 has also been found to be valid and reliable and had been used in a nationally representative survey to determine population estimates for prevalence, extent, and severity of impact on OHRQoL [20]. OHIP-14 assesses the burden of oral health status on life quality across seven conceptual domains (two items per domain) by asking respondents to rate the frequency of occurrence of a particular problem as captured by the individual item. The dimensions are: functional limitation, physical pain, psychological discomfort, physical disability, psychological disability, social disability, and handicap [21]. These dimensions are based on Locker's conceptual model of oral health [22]. Responses are rated on a 5 -point Likert scale: $0=$ never; $1=$ hardly ever; 2 = occasionally; 3 = fairly often; 4 = very often/ every day. Summary OHIP-14 scores can range from 0 to 56 and are calculated by summing the ordinal values for the 14 items. Domain scores can range from 0 to 8 . Higher OHIP-14 scores indicate worse, and lower scores indicate better, oral health-related quality of life. All participants completed the OHIP-14 before any orthodontic treatment.

\section{IOTN-DHC}

After participants were interviewed using OHIP-14, clinical examinations were conducted to assess normative orthodontic treatment need using the Dental Health Component (DHC) of the Index of Orthodontic Treatment Need (IOTN). This index has gained international acceptance 
because it is valid, reliable and easy to use [23]. For the Dental Health Component (DHC) of IOTN, 10 traits of malocclusion are assessed: overjet, reverse overjet, overbite, open bite, cross bite, crowding, impeded eruption, defects of cleft lip and palate as well as any craniofacial anomaly, Class II and Class III buccal occlusions, and hypodontia. Only the highest scoring trait is used to assess treatment need [24]. The treatment needs of the patients were categorized as Grade 1 (no treatment need), Grade 2 (little treatment need), Grade 3 (borderline need), and Grade 4 and 5 (high treatment need). Age, sex, and educational background were recorded because of their potential associations with both the outcome and explanatory variables.

\section{Examiner reliability}

IOTN-DHC ratings were recorded independently by two trained and calibrated examiners. To assess intra- and inter-examiner reliability, 20 young people who were not part of the present study were randomly selected and reexamined at a 2 to 4 week interval after their first examinations. Intra-examiner reliability for the IOTNDHC examiners was almost perfect with kappa $=0.91$ and 0.96. Excellent agreement was found for the interexaminer reliability with Kappa $=0.85$.

\section{Statistical analysis}

The data were analyzed by using R-Project software (version 2.13.2) [25]. Additive scale and subscale scores for the OHIP-14 were calculated by summing the item response codes. Data analyses included descriptive statistics and One-way ANOVA with Tukey Post Hoc test to assess differences in OHRQoL across groups defined by IOTNDHC. Bivariate and multivariate regression models were used to measure the association between OHRQoL and IOTN-DHC, sex, age and education level. In linear regression analysis, OHIP-14 scores were used as continuous outcome variable, IOTN-DHC was recoded as a series of dummy variables. Age was recoded into three categories, and dummy variables calculated for use in the regression analyses. Bivariate linear regressions were performed individually in separate models with each explanatory variable; model 1 included IOTN-DHC (No treatment required $=0$, little treatment required $=1$, borderline treatment required $=2$ and high treatment required $=3$ ), model 2 included gender $($ Male $=0$ and Female $=1)$, model 3 included age groups $(15-18$ years $=0,19-21$ years $=1,22-25$ years $=2)$ and model 4 included educational level (secondary education $=0$ and university education $=1$ ). Finally, all the explanatory variables were combined into a multivariate linear regression model.

\section{Results}

A total of three hundred and twenty five young adults participated in this study, two records were excluded from analysis due to missing values in their OHIP-14 questionnaires. The mean $( \pm$ SD) age was $22.05( \pm 3.20)$ years which included 63 participants (19.5\%) aged 15-18 years, 89 (27.5\%) aged 19-21 years and 171 (53.0\%) in the 22-25 age group. Most of the participants (273, $85.4 \%)$ had education to university level and 49 (15.1\%) were educated only to secondary level. Of the 323 participants, 132 (40.8\%) were boys and 191 (59.1\%) were girls. Normative treatment need according to IOTN-DHC was present in $252(78.0 \%)$ participants, the remaining 71 participants $(22 \%)$ did not have any normative treatment need for orthodontic treatment as assessed by the IOTN-DHC.

Table 1 displays the mean, standard deviation, median and the range for the total OHIP-14 score and for each domain in all 322 participants. The mean overall score ( \pm SD) for OHIP-14 was $22.6 \pm 12.5$. The psychological disability $(2.6 \pm 1.8)$ domain of the OHIP-14 showed the least impact due to malocclusion in patients with perceived need for malocclusion. Whereas, the psychological discomfort domain had the highest reported impact with a mean $( \pm$ SD) $4.0 \pm 1.9$.

Table 2 shows the results from the Oneway ANOVA and post hoc Tukey test comparing groups defined by IOTN-DHC. Participants with high treatment need reported a significantly greater negative impact on the overall OHRQoL score and in each domain of OHIP-14. The greatest impact was seen in the psychological discomfort domain where even having little treatment need was associated with a significant difference in OHIP-14 scores in comparison to the "No treatment need" group. Whereas, the domains of functional limitation, physical pain, physical disability, psychological disability and social disability all showed a significant difference in OHIP-14 score at the level of the "borderline treatment need" group, the handicap domain only showed a significant difference in OHIP-14 scores in the "high treatment need" group.

Table 3 shows the results of the bivariate and multivariate analyses to establish the association between

Table 1 Mean, standard deviation (SD), median and range observed in Oral Health Impact Profile- 14 (OHIP-14)

\begin{tabular}{lccc}
\hline OHIP domain & Mean \pm SD & Median & Range observed \\
\hline Functional limitation & $3.6 \pm 2.1$ & 4 & $0-8$ \\
Physical pain & $3.3 \pm 1.7$ & 4 & $0-7$ \\
Psychological discomfort & $4.0 \pm 1.9$ & 4 & $0-8$ \\
Physical disability & $3.2 \pm 2.0$ & 4 & $0-8$ \\
Psychological disability & $2.6 \pm 1.8$ & 2 & $0-7$ \\
Social disability & $2.7 \pm 2.1$ & 3 & $0-6$ \\
Handicap & $3.2 \pm 2.0$ & 4 & $0-6$ \\
OHIP-14 total & $22.6 \pm 12.5$ & 25 & $0-46$ \\
\hline
\end{tabular}


Table 2 Mean scores in overall and seven domains of Oral Health Impact Profile-14 (OHIP-14) OHIP-14 among different types of Index of Orthodontic Treatment Need- Dental Health Component (IOTN-DHC) groups

\begin{tabular}{|c|c|c|c|c|c|c|c|c|c|}
\hline IOTN-DHC ${ }^{1}$ & N (\%) & $\begin{array}{l}\text { Functional } \\
\text { limitation }\end{array}$ & $\begin{array}{l}\text { Physical } \\
\text { pain }\end{array}$ & $\begin{array}{l}\text { Psychological } \\
\text { discomfort }\end{array}$ & $\begin{array}{l}\text { Physical } \\
\text { disability }\end{array}$ & $\begin{array}{l}\text { Psychological } \\
\text { disability }\end{array}$ & $\begin{array}{l}\text { Social } \\
\text { disability }\end{array}$ & Handicap & $\begin{array}{l}\text { OHIP-14 } \\
\text { total }\end{array}$ \\
\hline $\begin{array}{l}\text { No treatment need } \\
\text { (Reference Group) }\end{array}$ & 71 (22.0\%) & $2.2 \pm 1.8$ & $2.1 \pm 1.6$ & $2.6 \pm 1.8$ & $2.0 \pm 1.6$ & $2.0 \pm 1.6$ & $1.8 \pm 1.6$ & $2.4 \pm 1.7$ & $15.2 \pm 10.9$ \\
\hline Little treatment need & $87(26.9 \%)$ & $3.2 \pm 1.9$ & $3.0 \pm 1.6$ & $3.8 \pm 1.8^{* * *}$ & $3.0 \pm 1.9$ & $2.0 \pm 1.6$ & $2.2 \pm 2.0$ & $2.9 \pm 1.8$ & $20.2 \pm 11.1^{*}$ \\
\hline Borderline treatment need & $80(24.8 \%)$ & $3.9 \pm 2.0^{* *}$ & $3.4 \pm 1.3^{* *}$ & $4.5 \pm 1.6^{* * *}$ & $3.4 \pm 1.9^{*}$ & $2.9 \pm 1.7^{*}$ & $2.9 \pm 1.9^{*}$ & $3.2 \pm 1.9$ & $24.1 \pm 11.2^{* *}$ \\
\hline High Treatment need & $85(26.3 \%)$ & $4.8 \pm 2.0^{* * *}$ & $4.2 \pm 1.7^{* * *}$ & $4.9 \pm 1.7^{* * *}$ & $4.0 \pm 2.0^{* * *}$ & $3.6 \pm 1.9^{* * *}$ & $3.8 \pm 2.2^{* * *}$ & $4.1 \pm 2.0^{* *}$ & $29.5 \pm 12.5^{* * *}$ \\
\hline
\end{tabular}

${ }^{*} \mathrm{P}<0.05$, ${ }^{* *} \mathrm{P}<0.01$ and ${ }^{* *} \mathrm{P}<0.001$.

${ }^{1}$ Group comparisons were performed by One Way Analysis of Variance (ANOVA) and Tukey Post Hoc test.

OHIP-14 and IOTN-DHC, gender, age group and education level. There is a significant association of normative orthodontic treatment need with overall OHIP-14 score $(\mathrm{p}<0.05)$. Although females have slightly higher impact scores than males, this is not significant in either analysis. Age group is negatively associated with overall OHIP-14 score $(\mathrm{p}<0.05)$. The $15-18$ year old group showed the highest impact on their quality of life due to malocclusion. This age group association becomes stronger in the multivariate model. The association between education level and IOTN was not significant in the bivariate model, but became significant in the multivariate model, participants with a university education report a significantly higher impact on OHRQoL as compared to participants with only secondary education. The final multivariate model explained $22 \%$ variation in the total score of OHIP-14 $\left(\mathrm{R}^{2}=0.22\right)$.

\section{Discussion}

This study evaluated the impact of orthodontic treatment need on overall OHRQoL and its various domains in 15-25 year olds seeking orthodontic treatment at UiTM Dental clinics. Nearly 1.5 times as many girls as boys sought orthodontic treatment at the university clinic during the recruitment period for this study, this agrees with previous studies which show higher

Table 3 Bivariate and multivariate linear regression models showing association of Oral Health Impact Profile-14 (OHIP-14) with Index of Orthodontic Treatment Need- Dental Health Component (IOTN-DHC) and other covariates

\begin{tabular}{|c|c|c|c|c|c|}
\hline & \multicolumn{3}{|c|}{ Bivariate analysis } & \multicolumn{2}{|c|}{ Multivariate analysis } \\
\hline & Estimates & SE & $\mathrm{R}^{2}$ & Estimates & SE \\
\hline \multicolumn{6}{|l|}{ IOTN-DHC } \\
\hline Intercept & 15.239 & $1.37^{* * *}$ & 0.16 & & \\
\hline No treatment need & \multicolumn{2}{|c|}{ Reference Group } & & \multicolumn{2}{|c|}{ Reference Group } \\
\hline Little treatment need & 4.97 & $1.84^{* *}$ & & 4.77 & $1.79^{* *}$ \\
\hline Borderline treatment need & 8.88 & $1.88^{* * *}$ & & 9.03 & $1.83^{* * *}$ \\
\hline High Treatment need & 14.27 & $1.85^{* * *}$ & & 14.41 & $1.80^{* * *}$ \\
\hline \multicolumn{6}{|l|}{ Sex } \\
\hline Intercept & 21.9 & $0.90^{* * *}$ & 0.003 & & \\
\hline Male & \multicolumn{2}{|c|}{ Reference Group } & & \multicolumn{2}{|c|}{ Reference Group } \\
\hline Female & 1.47 & 1.41 & & 1.36 & 1.28 \\
\hline \multicolumn{6}{|l|}{ Age } \\
\hline Intercept & 27.06 & $1.55^{* * *}$ & 0.03 & & \\
\hline $15-18$ years & \multicolumn{2}{|c|}{ Reference Group } & & \multicolumn{2}{|c|}{ Reference Group } \\
\hline $19-21$ years & -4.01 & $2.03^{*}$ & & -6.12 & $2.22^{* *}$ \\
\hline $22-25$ years & -6.40 & $1.81^{* * *}$ & & -9.46 & $2.12^{* * *}$ \\
\hline \multicolumn{6}{|l|}{ Education Level } \\
\hline Intercept & 23.44 & $1.7^{* * *}$ & 0.009 & & \\
\hline Secondary education & \multicolumn{2}{|c|}{ Reference Group } & & \multicolumn{2}{|c|}{ Reference Group } \\
\hline \multirow[t]{2}{*}{ University education } & -1.05 & 1.9 & & 4.45 & $2.24^{*}$ \\
\hline & & & & \multicolumn{2}{|c|}{$R^{2}=0.22$} \\
\hline
\end{tabular}

${ }^{*} \mathrm{P}<0.05$, ${ }^{* *} \mathrm{P}<0.01$ and ${ }^{* * *} \mathrm{P}<0.001$. 
utilization of orthodontic treatment services by females $[3,15,26,27]$. This may suggest that women are responding to social expectations of the importance of esthetics rather than an objectively greater orthodontic treatment need in comparison to their male counterparts [28]. Alternatively parents may be more prone to seek orthodontic treatment for their female children than their male. It was expected that the impact of malocclusion on quality of life would be significantly greater in girls as compared to boys, since boys may be less self-conscious about their appearance [28]. Young women were more likely to have had a higher dental impact than male youths but the difference was not significant. Similar findings were reported by Oliveira and Sheiham (29) in adolescents [29] and other age groups by Birkeland et al., Hunt et al., 57 and Bernabe 'et al. [8,30,31]. However, Peres (2008) et al. found females adolescents had greater dissatisfaction with their dental appearance [32]. It is possible that the lack of a difference in impact between males and females in this and other studies is due to selection: the reluctance of males to attend clinics resulting in those who feel little or no impact not attending.

Bivariate and multivariate regression analysis showed that those participants with more severe malocclusions reported a greater impact on quality of life after controlling for the effects of covariates (sex, age, and education level). Young people with little, borderline and high orthodontic treatment need had 5, 9 and 15 points higher scores on the OHIP-14 scale, respectively, when compared to the "no treatment need" group. This relationship between malocclusions and OHRQOL mirrors that found by Heravi et.al., Bernabe et. al. and Foster-Page et. al. $[8,14,28]$, but not by Taylor et al. or Oliveria et al. (2008) $[16,33]$. There are several possible reasons for such differences. First, the use of different measures to measure quality of life. Secondly it is possible that different age groups have a different perception towards esthetics and quality of life, and studies have varied widely in the age groups studied. In addition, different cultures, traditions, and social norms across countries may influence the perception of esthetics different in each society. Finally, a high frequency or severity of malocclusions in some races and ethnic groups can make malocclusion perceived as normal for the given group and vice versa [28].

A negative association was observed between age and impact on quality of life due to malocclusion, the impact of malocclusion decreases as age increases. This may be the result of 'response shift' with age - the longer an individual lives with a malocclusion, the greater the likelihood that they will adjust to the limitations it places upon their activities thereby reducing impact. In order to explore this, future studies should look at treatment need changes with age. Education level had a positive association in the multivariate regression analysis which may be due to increased self awareness and self esteem with increasing education.
Exploring each OHIP-14 domain, IOTN scores were most closely correlated with impact on the psychological discomfort and functional limitation domains of OHRQoL. Similar results have been reported in children aged $11-14$ years $[4,14]$ and in young adults $[3,29]$. This is logical when we consider that the most common reason for seeking orthodontic treatment is to correct dental esthetics and improve self-esteem [3]. Thus, orthodontists should be aware that young patients might expect orthodontic treatment to provide not only improved oral functioning and health but enhancement of esthetics, selfesteem and social life [34]. When these expectations are not met this may lead to dissatisfaction with treatment outcome. The use of the OHRQoL measure as a part of the diagnostic procedure can provide information on priorities for treatment in order to maximize patient satisfaction $[3,12,35]$. In this study, significant impact on the handicap domain was only present when patients had high treatment need. Some authors have suggested that malocclusion might become handicapping not because of the functional disability, but because it can adversely affect social relationships and self-perceptions [36].

The findings of this study must be tempered by a consideration of its limitations. The participants in this study were 15-25 year-old patients seeking orthodontic treatment at a university clinic in a large urban city in Malaysia. Therefore the results from this study cannot be extrapolated to the entire youth population who may have differing levels of malocclusion and orthodontic treatment needs and, therefore, different impacts on their daily activities [12]. Although many studies have assessed the impact of oral conditions on children's quality of life using convenience samples in hospitals or universities $[1,4,14]$, future studies should be based on a representative sample with or without normative need. Furthermore participants may have exaggerated the impact in order to increase their chances to obtain treatment for their malocclusion.

OHIP-14 and IOTN-DHC were used in this study, both of these instruments are valid and reliable but have some limitations [3]. IOTN may be a relatively insensitive instrument to measure minor occlusal traits and irregularities which mostly affect patient appearance and about which a patient is deeply concerned $[8,10]$. The OHIP-14 was developed for adults, but has been successfully applied to adolescents by many authors $[3,27,29,37$ because adolescents of 12 years of age and above are capable of abstract thinking, reasoning about the timing of past events, and relating them with good or bad experiences [3]. Another limitation with OHIP-14 is, it does not elicit the specific cause(s) of the impacts recorded, which can be related to a variety of oral health conditions and not necessarily the subject's malocclusion. However the participants in this study were selected to be free of untreated caries, periodontal disease and any other oral 
health problem suggesting the results form OHIP-14 were not confounded with other oral health condition.

\section{Conclusion}

Malocclusion has a negative impact on oral health related quality of life and its domains, this is highest for the psychological discomfort domain. Reported impact is greatest in younger people and those with a university education, whereas this study did not find any significant difference in the impact on oral health related quality of life in male and females.

\section{Competing interests}

The authors declare that they have no competing interests.

\section{Authors' contributions}

All the authors approved the final draft of the manuscript. YM contributed to data analysis and manuscript writing; NBZ and NBA contributed to participants recruitment and data collection. SFH helped in malocclusion data collection. TN and $\mathrm{MM}$ contributed to the conceptual framework of the project.

\section{Author details}

${ }^{1}$ Centre of Studies for Oral \& Maxillofacial Diagnostic and Medicine, Faculty of Dentistry, Universiti Teknologi MARA, Shah Alam, Malaysia. ${ }^{2}$ Centre of Studies for Community Dentistry, Faculty of Dentistry, Universiti Teknologi MARA, Shah Alam, Malaysia. ${ }^{3}$ Faculty of Dentistry, Universiti Teknologi MARA, Shah Alam, Malaysia. ${ }^{4}$ Centre of Studies for Orthodontics, Faculty of Dentistry, Universiti Teknologi MARA, Shah Alam, Malaysia. ${ }^{5}$ Unit of Dental Public Health and Oral Health Services Research, King's College Dental Institute, King's College London, Denmark Hill, London, UK.

Received: 25 September 2012 Accepted: 28 December 2012 Published: 26 February 2013

\section{References}

1. Aldrigui JM, Abanto J, Carvalho TS, Mendes FM, Wanderley MT, Bonecker M, Raggio DP: Impact of traumatic dental injuries and malocclusions on quality of life of young children. Health Qual Life Outcomes 2011, 9:78.

2. Masood M, Masood Y, Saub R, Newton J: Need of minimal important difference for oral health-related quality of life measures. J Public Health Dent 2012.

3. Feu D, de Oliveira BH, de Oliveira Almeida MA, Kiyak HA, Miguel JA: Oral healthrelated quality of life and orthodontic treatment seeking. Am J Orthod Dentofacial Orthop 2010, 138:152-159.

4. O'Brien C, Benson PE, Marshman Z: Evaluation of a quality of life measure for children with malocclusion. J Orthod 2007, 34:185-193. discussion 176.

5. Petersen PE, Bourgeois D, Ogawa H, Estupinan-Day S, Ndiaye C: The global burden of oral diseases and risks to oral health. Bull World Health Organ 2005, 83:661-669.

6. Rusanen J, Lahti S, Tolvanen M, Pirttiniemi P: Quality of life in patients with severe malocclusion before treatment. Eur J Orthod 2010, 32:43-48.

7. Onyeaso $\mathrm{CO}$ : Orthodontic treatment complexity and need with associated oral health-related quality of life in Nigerian adolescents. Oral Health Prev Dent 2009, 7:235-241.

8. Bernabe E, Sheiham A, Tsakos G, Messias de Oliveira C: The impact of orthodontic treatment on the quality of life in adolescents: a case-control study. Eur J Orthod 2008, 30:515-520.

9. de Oliveira CM, Sheiham A: The relationship between normative orthodontic treatment need and oral health-related quality of life. Community Dent Oral Epidemiol 2003, 31:426-436.

10. De Baets E, Lambrechts H, Lemiere J, Diya L, Willems G: Impact of self-esteem on the relationship between orthodontic treatment need and oral health-related quality of life in 11- to 16-year-old children. Eur J Orthod 2012, 34:731-737.

11. Kok YV, Mageson P, Harradine NW, Sprod AJ: Comparing a quality of life measure and the Aesthetic Component of the Index of Orthodontic Treatment Need (IOTN) in assessing orthodontic treatment need and concern. J Orthod 2004, 31:312-318. discussion 300-311.

12. Johal A, Cheung MY, Marcene W: The impact of two different malocclusion traits on quality of life. Br Dent J 2007, 202:E2.
13. Agou S, Malhotra M, Tompson B, Prakash P, Locker D: Is the child oral health quality of life questionnaire sensitive to change in the context of orthodontic treatment? A brief communication. J Public Health Dent 2008, 68:246-248.

14. Foster Page LA, Thomson WM, Jokovic A, Locker D: Validation of the Child Perceptions Questionnaire (CPQ 11-14). J Dent Res 2005, 84:649-652.

15. Locker D, Jokovic A, Clarke M: Assessing the responsiveness of measures of oral health-related quality of life. Community Dent Oral Epidemiol 2004 32:10-18.

16. de Oliveira CM, Sheiham A, Tsakos G, O'Brien KD: Oral health-related quality of life and the IOTN index as predictors of children's perceived needs and acceptance for orthodontic treatment. Br Dent J 2008, 204:1-5. discussion 384-385.

17. Zhang M, McGrath C, Hagg U: The impact of malocclusion and its treatment on quality of life: a literature review. Int J Paediatr Dent 2006, 16:381-387.

18. Allen PF, McMillan AS, Walshaw D, Locker D: A comparison of the validity of generic- and disease-specific measures in the assessment of oral healthrelated quality of life. Community Dent Oral Epidemiol 1999, 27:344-352.

19. Slade GD: Derivation and validation of a short-form oral health impact profile. Community Dent Oral Epidemiol 1997, 25:284-290.

20. Saub R, Locker D, Allison P: Derivation and validation of the short version of the Malaysian Oral Health Impact Profile. Community Dent Oral Epidemio 2005, 33:378-383.

21. Slade GD, Spencer AJ: Development and evaluation of the Oral Health Impact Profile. Community Dent Health 1994, 11:3-11.

22. Locker D: Measuring oral health: a conceptual framework. Community Dent Health 1988, 5:3-18.

23. Jarvinen S: Indexes for orthodontic treatment need. Am J Orthod Dentofacial Orthop 2001, 120:237-239.

24. Brook PH, Shaw WC: The development of an index of orthodontic treatment priority. Eur J Orthod 1989, 11:309-320.

25. Team R: R: A Language and Environment for Statistical Computing. Vienna: Austria; 2012

26. Al Yami EA, Kuijpers-Jagtman AM, van't Hof MA: Orthodontic treatment need prior to treatment and 5 years postretention. Community Dent Oral Epidemiol 1998, 26:421-427.

27. Broder $\mathrm{HL}$, Slade G, Caine R, Reisine S: Perceived impact of oral health conditions among minority adolescents. J Public Health Dent 2000, 60:189-192.

28. Heravi F, Farzanegan F, Tabatabaee M, Sadeghi M: Do malocclusions affect the oral health-related quality of life? Oral Health Prev Dent 2011, 9:229-233.

29. de Oliveira CM, Sheiham A: Orthodontic treatment and its impact on oral health-related quality of life in Brazilian adolescents. J Orthod 2004, 31:20-27. discussion 15.

30. Birkeland K, Boe OE, Wisth PJ: Subjective assessment of dental and psychosocial effects of orthodontic treatment. J Orofac Orthop 1997, 58:44-61.

31. Hunt O, Hepper P, Johnston C, Stevenson M, Burden D: The aesthetic component of the index of orthodontic treatment need validated against lay opinion. Eur J Orthod 2002, 24:53-59.

32. Peres KG, Barros AJ, Anselmi L, Peres MA, Barros FC: Does malocclusion influence the adolescent's satisfaction with appearance? A crosssectional study nested in a Brazilian birth cohort. Community Dent Oral Epidemiol 2008, 36:137-143.

33. Tonelli R, Mclntyre A, Camerin C, Walters ZS, Di Leo K, Selfe J, Purgato S, Missiaglia E, Tortori A, Renshaw J, et al: Antitumor activity of sustained Nmyc reduction in rhabdomyosarcomas and transcriptional block by antigene therapy. Clin Cancer Res 2012, 18:796-807.

34. Tung AW, Kiyak HA: Psychological influences on the timing of orthodontic treatment. Am J Orthod Dentofacial Orthop 1998, 113:29-39.

35. Agou S, Locker D, Muirhead V, Tompson B, Streiner DL: Does psychological well-being influence oral-health-related quality of life reports in children receiving orthodontic treatment? Am J Orthod Dentofacial Orthop 2011, 139:369-377.

36. Prahl-Andersen B: The need for orthodontic treatment. Angle Orthod 1978, 48:1-9.

37. Thomas CW, Primosch RE: Changes in incremental weight and well-being of children with rampant caries following complete dental rehabilitation. Pediatr Dent 2002, 24:109-113.

doi:10.1186/1477-7525-11-25

Cite this article as: Masood et al: Impact of malocclusion on oral health related quality of life in young people. Health and Quality of Life Outcomes 2013 11:25. 